\title{
Rare FOXC1 variants in congenital glaucoma: identification of translation regulatory sequences
}

\author{
Cristina Medina-Trillo ${ }^{1,2}$, José-Daniel Aroca-Aguilar ${ }^{1,2}$, Carmen-Dora Méndez-Hernández ${ }^{2,3}$, Laura Morales ${ }^{2,3}$, \\ Maite García-Antón ${ }^{2,3,4}$, Julián García-Feijoo ${ }^{2,3}$ and Julio Escribano ${ }^{\star, 1,2}$
}

Primary congenital glaucoma (PCG) is the cause of a significant proportion of inherited visual loss in children, but the underlying mechanism is poorly understood. In this study, we assessed the relationship between PCG and FOXC1 variants by Sanger sequencing the proximal promoter and transcribed sequence of FOXC1 from a cohort of 133 PCG families with no known CYP1B1 or MYOC mutations. The pathogenicity of the identified variants was evaluated by functional analyses. Ten patients (7.5\%) with no family history of glaucoma carried five different rare heterozygous FOXC1 variants with both increased (rs77888940:C > G, c.-429C > G, rs730882054:c.1134_144del(CGGCGGCGCGG), p.(G380Rfs*144) and rs35717904:A $>$ T, c. ${ }^{*} 734 \mathrm{~A}>\mathrm{T}$ ) and decreased (rs185790394: C $>$ T, c. $-244 \mathrm{C}>\mathrm{T}$ and rs79691946:C $>$ T, p.(P297S)) transactivation, ranging from 50 to $180 \%$ of the wild-type activity. The five variants did not show monogenic segregation, and four of them were absent in a control group $(n=233)$. To the best of our knowledge, one of these variants (p.(G380Rfs*144)) has not previously been described. One of the FOXC1 variant carriers (p.(P297S)) also coinherited a functionally altered rare PITX2 heterozygous variant ( $r$ 6533526:C $>\mathrm{T}, \mathrm{c} .{ }^{*} 454 \mathrm{C}>\mathrm{T}$ ). Bioinformatics and functional analyses provided novel information on three of these variants. c. $-429 C>G$ potentially disrupts a consensus sequence for a terminal oligopyrimidine tract, whereas $c .-244 C>T$ may alter the RNA secondary structure in the $5^{\prime}$-untranslated region (UTR) that affects mRNA translation. In addition, p.(G380Rfs*144) led to increased protein stability. In summary, these data reveal the presence of translation regulatory sequences in the UTRs of FOXC1 and provide evidence for a possible role of rare FOXC1 variants as modifying factors of goniodysgenesis in PCG. European Journal of Human Genetics (2016) 24, 672-680; doi:10.1038/ejhg.2015.169; published online 29 July 2015

\section{INTRODUCTION}

Primary congenital glaucoma (PCG; MIM\# 231300) is the most common form of childhood glaucoma and represents a significant cause of visual loss and blindness in children. PCG results from developmental abnormalities of the anterior segment structures of the eye that are required for aqueous humor $(\mathrm{AH})$ drainage. These abnormalities lead to an increase in $\mathrm{AH}$ outflow resistance, elevated intraocular pressure (IOP) and optic nerve degeneration. The PCG incidence varies between different populations, with an estimated overall occurrence of approximately 1 in 10000 births. PCG is genetically heterogeneous. Four PCG loci have been mapped (GLC3A at $2 \mathrm{p} 21, G L C 3 B$ at $1 \mathrm{p} 36, G L C 3 C$ at $14 \mathrm{q} 24.3$ and GLC3D at $14 \mathrm{q} 24.3)$, although only two glaucoma genes have been identified, cytochrome P450, subfamily I, polypeptide 1 (CYP1B1 MIM\# 601771) and latent transforming growth factor- $\beta$-binding protein 2 (LTBP2 MIM\# 602091), situated on the GLC3A and the GLC3D loci, respectively. In previous studies we showed that $\sim 30 \%$ of Spanish PCG patients carry loss-offunction $C Y P 1 B 1$ variants, with most of these variants resulting in null genotypes. ${ }^{1}$ Interestingly, the existence of incomplete penetrance, variable expressivity and the fact that a significant proportion of patients carry nondominant heterozygous CYP1B1 mutations ${ }^{1}$ suggest that more than one gene can be involved in PCG inheritance. Mutations in LTBP2, ${ }^{2}$ MYOCILIN (MYOC, MIM\# 601652) (3,4 $^{3,4}$ forkhead box C1 (FOXC1 MIM\# 601090) have also been found in a small number of Indian PCG cases. ${ }^{5}$

FOXC1 mutations have been found to be involved in several dominant ocular defects, including Axenfeld-Rieger syndrome type 3 (ARS; MIM\# 602482), a disease that is associated with increased glaucoma risk and variability in iris and extraocular abnormalities. ${ }^{6}$ The FOXC1 gene encodes a transcription factor that regulates craniofacial, cardiovascular and ocular development. ${ }^{7}$ The FOXC1 protein belong to the FOX class of transcription factors and is characterized by a conserved 110 amino-acid DNA-binding domain, called the forkhead domain (FHD). FOXC1 is present in mesoderm and neural crest-derived cells, including cells of the anterior segment of the eye, and the periocular mesenchyme and mesenchimal cells that migrate into the eye.

In this study, we show the existence of regulatory signals in the untranslated regions (UTRs) of FOXC1 that are involved in downregulating its translation, and we provide evidence for the putative role of rare functionally altered FOXC1 variants as modifying factors in PCG.

\section{MATERIALS AND METHODS}

Subjects

A total of 133 unrelated families affected by PCG were included in this study. The study and informed consent procedures were approved by the Ethics

${ }^{1}$ Área de Genética, Facultad de Medicina/Instituto de Investigación en Discapacidades Neurológicas (IDINE), Universidad de Castilla-La Mancha, Albacete, Spain; ${ }^{2}$ Cooperative Research Network on Age-Related Ocular Pathology, Visual and Life Quality, Instituto de Salud Carlos III, Madrid, Spain; ${ }^{3}$ Servicio de Oftalmología, Hospital San Carlos, SPAIN/ Instituto de Investigación Sanitaria del Hospital Clínico San Carlos, Madrid, Spain; ${ }^{4}$ Instituto de Investigaciones Oftalmológicas Ramón Castroviejo, Facultad de Medicina, Universidad Complutense de Madrid, Madrid, Spain

*Correspondence: Professor J Escribano, Área de Genética, Facultad de Medicina/Instituto de Investigación en Discapacidades Neurológicas (IDINE), Universidad de Castilla-La Mancha, Avda de Almansa 14, Albacete 02006, Spain. Tel: +34 967599200 ; Fax: +34 902 204130; E-mail: julio.escribano@uclm.es

Received 20 November 2014; revised 19 June 2015; accepted 25 June 2015; published online 29 July 2015 
Committee for Human Research of the Hospital Clínico San Carlos, Madrid (Spain), and followed the tenets of the Declaration of Helsinki. Informed written consent was obtained from all of the participants and was recorded by staff involved in the study.

All subjects were clinically evaluated by glaucoma specialists. The ophthalmic examination included slit lamp biomicroscopy, gonioscopy, biometry, IOP measurement and ophthalmoscopy. The PCG clinical diagnosis was performed as previously described. ${ }^{1}$ Patients over 3 years of age who were diagnosed with abnormal gonioscopy of the anterior segment (high iris insertion and absence of an angle recess), were considered to be late congenital glaucoma (LCG) cases. Patients with pediatric glaucoma that was either related to other ocular disorders or associated with systemic abnormalities were excluded. Ocular and nonocular features of ARS were ruled out in the patients. Control individuals were recruited from among those who attended the clinic for conditions other than glaucoma, including cataracts, floaters, refractive errors and itchy eyes. They also underwent full ocular exploration, including IOP measurement, determination of best visual acuity with optical correction, gonioscopy and eye fundus examination.

\section{Variant screening}

The genomic DNA was extracted from peripheral blood, using the QIAamp DNA Blood Mini Kit (Qiagen, Valencia, CA, USA). The DNA sequence variation analyses were carried out by automatic Sanger sequencing. A fragment of the promoter region (nucleotides -1 to -875 ), the coding region and the $5^{\prime}$ - and $3^{\prime}$-UTRs of FOXC1, and the PITX2 coding region and its 5'- and $3^{\prime}$-UTRs were amplified by PCR, using the primers described in Supplementary Table 1 . The variants identified in each subject were confirmed by sequencing a new amplification product. The rs35717904:A > T, c. ${ }^{\star} 734 \mathrm{~A}>\mathrm{T}$ FOXC1 variant was genotyped in controls by Taqman SNP genotyping assay (4351379, Applied Biosystems, Foster City, CA, USA). The entire MYOC and CYP1B1 coding regions were checked in all patients for disease-causing variants by Sanger DNA sequencing as previously described. ${ }^{8,9}$

\section{Site-directed mutagenesis and cloning of the FOXC1 and PITX2 variants}

Two FOXC1 variants (rs79691946:C > T, p.(P297S) and rs35717904:A > T, c. ${ }^{*} 734 \mathrm{~A}>\mathrm{T}$ ), one PITX2 nucleotide substitution (rs6533526:C $>\mathrm{T}$, c. ${ }^{\star} 454 \mathrm{C}>\mathrm{T}$ ) identified in this study and the control p.(I126M) FOXC1 variant were obtained by site-directed mutagenesis using the QuickChange SiteDirected Mutagenesis Kit (Stratagene, La Jolla, CA, USA), with the primers and PCR conditions indicated in the Supplementary Materials and methods. Variants p.(G380Rfs $\left.{ }^{\star} 144\right)$, c.1359_1361dup (p.(G456dup)), rs185790394:C > T, c. $-244 \mathrm{C}>\mathrm{T}$ and rs77888940:C $>$ G, c. $-429 \mathrm{C}>\mathrm{G}$ were also obtained as indicated in the Supplementary Materials and methods section. The recombinant FOXC1 and PITX2 versions were transiently expressed in human embryonic kidney 293 T (HEK-293 T) cells that were cultured as indicated in the Supplementary Materials and methods section. Transient plasmid transfections were carried out with 50-500 ng of plasmid DNA using the Superfect Transfection Reagent (Qiagen) according to the manufacturer's instructions.

\section{Luciferase assays}

The FOXC1 transactivation assays were performed using the Luciferase Assay System (Promega, Madison, WI, USA) according to the manufacturer's instructions. HEK-293 T cells were transfected with $500 \mathrm{ng}$ of the recombinant FOXC1 expression vector (pcDNA3.1), along with $50 \mathrm{ng}$ of the recombinant pGL3-basic-CXCR4 luciferase reporter, and $50 \mathrm{ng}$ of the pMirTarget vector as indicated in the Supplementary Materials and methods section. Finally, the cells were assayed for firefly luciferase activity.

To analyze the functional effect of the PITX2 rs6533526:C > T, c. ${ }^{*} 454 \mathrm{C}>\mathrm{T}$ variant, the mutant and wild-type $737 \mathrm{bp}$ of the $3^{\prime}$-UTR sequence (NM_000325) PITX2 were fused to the 3'-end of the firefly luciferase coding region (cloned into the pMirTarget vector; PS100062, OriGene, Technologies, Inc., Rockville, MD, USA). HEK-293 T cells were transfected with either the wild-type or the mutant $3^{\prime}$-UTR sequence, and $48 \mathrm{~h}$ later luciferase activity was measured.

\section{Protein stability and half-life calculation}

The protein stability was studied via western blot of transfected cells treated with cycloheximide $(300 \mu \mathrm{g} / \mathrm{ml})$ at different times as previously reported. ${ }^{9}$ The FOXC1 protein levels were determined by densitometry and the relative amounts at different time points after cycloheximide treatment were expressed as a percentage of the levels at time $0 \mathrm{~h}$. At least three independent assays for each variant were performed. The transfection efficiency was assessed by co-transfecting the recombinant cDNA constructs encoding the different mutants $(500 \mathrm{ng})$ with the pMirTarget vector $(50 \mathrm{ng})$ that encodes the red fluorescent protein (RFP). The RFP was detected via western blotting. The loaded samples were normalized for total protein content using the Bradford reagent (Pierce, Rockford, IL, USA). FOXC1 decay followed first-order kinetics. The slope of the decay line was calculated using standard linear regression, and the protein half-life was determined as previously described. ${ }^{10}$

\section{Western blotting and antibodies}

For the western blot analyses, the nuclear extracts, prepared as indicated in the Supplementary Materials and methods, were fractionated using sodium dodecyl sulfate-polyacrylamide gel electrophoresis (SDS-PAGE), using the MiniPROTEAN III Gel Electrophoresis System (Bio-Rad, Hercules, CA, USA) as previously described. ${ }^{11}$ A commercial mouse monoclonal anti-myc (Santa Cruz Biotechnology, Santa Cruz, CA, USA) was used as the primary antibody, diluted at $1: 1000$. Horseradish peroxidase-conjugated antibodies against mouse $\mathrm{IgG}$ (Pierce) were diluted to 1:1000-1:4000. Chemiluminiscence detection and the densitometry for protein band quantification was performed as previously described. ${ }^{11}$ As an additional sample loading control, the endogenous lactate dehydrogenase (LDH) protein was detected in cell extracts using a goat antiLDH antibody diluted to 1:5000 (AB1222, Chemicon, Temecula, CA, USA) and an anti-goat IgG horseradish peroxidase-conjugated antibody (sc-2033, Santa Cruz Biotechnology, diluted to 1:2000). RFP (transfection control) was detected using a rabbit anti-RFP antibody (AB233, Evrogen, Moscow, Russia), diluted to 1:5000, and an anti-rabbit IgG horseradish peroxidase-conjugated antibody (1858415, Pierce), diluted to 1:1000.

\section{Bioinformatic analyses}

The in silico analyses of FOXC1 and PITX2 sequences and their different variants were carried out using the programs described in Supplementary Materials and methods. Variants were named using directions from Mutalyzer (https://humgenprojects.lumc.nl/trac/mutalyzer), according to RefSeq NM_001453.2 and NM_00325.5 for FOXC1 and PITX2, respectively. The FOXC1 transcription start site and $5^{\prime}$-UTR sequence were defined as previously described. ${ }^{12}$ The first nucleotide of the translation initiation site was numbered as nucleotide +1 . All variants have been submitted to dbSNP (http://www.ncbi.nlm.nih.gov/SNP/).

\section{Statistical analysis}

The significance of the difference in sex and variant proportions between patients and control subjects was determined by either the $\chi^{2}$ test or Fisher's exact test when frequencies were below five. The statistical comparisons between groups were performed using either the $t$-test or the one-way analysis of variance (ANOVA). A Bonferroni correction was applied to adjust tests for multiple comparisons. The data were statistically processed by the SigmaStat 2.0 software (SPSS Science Inc., Chicago, IL, USA).

\section{RESULTS}

\section{Clinical phenotype of patients}

To analyze the role of FOXC1 variants in PCG, we studied 133 apparently unrelated PCG families. The main clinical features are presented in Table 1. The age of PCG diagnosis ranged from birth to 36 months, $12.0 \%$ of these cases were diagnosed at birth and $14.2 \%$ before the first month of life, and $73.8 \%$ were diagnosed between months 2 and 36 of life. Consanguinity was documented in only one family (PCG-148). The majority of cases were of Spanish ancestry, although six were immigrants from European or Arab countries. Three probands had mixed European origins of Spanish-German 
(PCG-43 and PCG-132) or Spanish-Italian (PCG-112). None of the patients carried disease-causing variants in the coding regions of $M Y O C$ and CYP1B1.

\section{FOXC1 variants}

Five different rare heterozygous sequence variants (Table 2) were identified in 10 of the 133 probands (7.5\%). The rs185790394: $\mathrm{C}>\mathrm{T}$, c. $-244 \mathrm{C}>\mathrm{T}$ variant was identified in five probands in the heterozygous state, but additional familial samples for segregation analysis were only available for two of those families (PCG-12 and PCG-14, Figure 1a). The fathers of these two patients (PCG-12 and PCG-14) were heterozygous carriers of the c. $-244 \mathrm{C}>\mathrm{T}$ variant and manifested elevated IOP, but no other ocular or visual alterations were observed at the time of the study (PCG-12, I:1 and PCG-14, I:1; Figure 1a and Table 2). Two other index cases (PCG-96 and PCG-169) were heterozygous carriers for the rs77888940:C $>$ G, c. $-429 \mathrm{C}>\mathrm{G}$ variant. The first patient showed bilateral glaucoma and inherited the nucleotide change from a healthy mother (Figure 1b), and the second carrier presented unilateral glaucoma (PCG-169, Table 2). The two variants present in these patiens are listed in the Ensembl variation database, but no population frequency data are available. The

Table 1 Clinical and demographic features of the patients included in this study

\begin{tabular}{lc}
\hline Variable & $P C G(n=133)$ \\
\hline Age (mean \pm SD) & 12.6 months \pm 20.1 \\
Female (\%) & 38.0 \\
Male (\%) & 62.0 \\
C/D/eye (mean \pm SD) & $0.53 \pm 0.27$ \\
IOP (mm Hg)/eye (mean \pm SD) & $26.3 \pm 9.9$ \\
Bilateral PCG (\%) & 73.9 \\
Unilateral PCG (\%) & 26.1 \\
Immigrants (\%) & 4.5 \\
\hline
\end{tabular}

Abbreviations: $C / D$, cup/disc ratio of optic nerve at diagnosis; IOP, intraocular pressure.
rs35717904:A $>\mathrm{T}$, c. ${ }^{\star} 734 \mathrm{~A}>\mathrm{T}$ variant was identified in a subject diagnosed with glaucoma in only one eye (Figure 1c). This nucleotide change has been described at low frequencies in Europeans (Ensembl variation database). rs730882054:c.1134_144delCGGCGGCGCGG, p. (G380Rfs $\left.{ }^{\star} 144\right)$ was present in the PCG-54 proband (Figure 1d), who inherited it from his asymptomatic mother. This nucleotide deletion was considered to be novel because it was not present in the Emsembl variation database, the Human Gene Mutation Database ${ }^{13}$ (http://www.hgmd.cf.ac.uk/ac/index.php), GeneCards (www.genecards.org), Online Mendelian Inheritance in Man (OMIM, http:// omim.org/) or PubMed (http://www.ncbi.nlm.nih.gov/pubmed). In addition, c.1359_1361dup, p.(G456dup) (described as rs572346201: c.1337_1338insCGG, p.(G456_Q457insG) in dbSNP) was present in three of these patients (PCG-96, PCG-98 and PCG-54) in the heterozygous state. Pedigree analyses of the available family members did not support the involvement of all the identified variants in monogenic glaucoma transmission. All rare variants were genotyped in 233 Spanish control individuals (466 chromosomes) and only c. $-244 \mathrm{C}>\mathrm{T}$ was identified in two control subjects in heterozygosis (allelic frequency $0.4 \%$ ).

\section{Coinheritance of rare FOXC1 and PITX2 variants}

Analyses of PITX2 sequence in the carriers of rare FOXC1 variants led to identification of the rs6533526:C $>\mathrm{T}, \quad c^{*} 454 \mathrm{C}>\mathrm{T}$ nucleotide substitution in the heterozygous state in one patient (PCG-84) (Figure 1e). This subject was a double heterozygote for FOXC1 and PITX2 variants (rs79691946:C > T, p.(P297S)/+ and c. ${ }^{*} 454 \mathrm{C}>\mathrm{T} /+$, respectively) and was diagnosed with bilateral glaucoma at the age of 6 months. Among the three nonaffected siblings of the proband, subject II:3 harbored one copy of the PITX2 variant, subject II:4 had the wild-type genotype for both nucleotide substitutions and subject II:5 had one copy of the FOXC1 variant (Figure 1e). Finally, the proband's 2 year-old daughter (III:1) also inherited both the FOXC1 and PITX2 variants, and was asymptomatic at the time of the study (2 years, Table 2). p.(P297S) has previously been found in the heterozygous state in patients with microphthalmia and anterior

Table 2 Rare FOXC1 and PITX2 gene variations identified in PCG and associated clinical features

\begin{tabular}{|c|c|c|c|c|c|c|c|}
\hline $\begin{array}{l}\text { Proband (family } \\
\text { member) }\end{array}$ & Nucleotide change ${ }^{a}$ & $\begin{array}{l}\text { Amino-acid } \\
\text { change }\end{array}$ & $\begin{array}{c}\text { Phenotype/ } \\
\text { laterality }\end{array}$ & $\begin{array}{l}\text { Age at diagnosis } \\
\text { (months)/sex }\end{array}$ & $\begin{array}{c}\text { IOP }(\mathrm{mm} \mathrm{Hg}) \text { at diagno- } \\
\text { sis }(O D / O \mathrm{I})\end{array}$ & $C / D(O D / O S)$ & Treatment $^{\mathrm{b}}$ \\
\hline PCG-10 & c. $-244 C>T$ & - & PCG/B & $9 / F$ & $(30 / 30)$ & NA & Surgery \\
\hline PCG-12 & c. $-244 C>T$ & - & PCG/B & $1.5 / \mathrm{M}$ & $(22 / 24)$ & $0.3 / 0.7$ & Surgery \\
\hline PCG-12 (I:1) & c. $-244 C>T$ & - & $\mathrm{OH} / \mathrm{B}$ & 708/M & $24 / 24$ & $0.5 / 0.5$ & NA \\
\hline PCG-14 & c. $-244 C>T$ & - & PCG/B & $1 / \mathrm{M}$ & $(22 / 22)$ & $0.3 / 0.5$ & Surgery \\
\hline PCG-14 (I:1) & c. $-244 C>T$ & - & $\mathrm{OH} / \mathrm{B}$ & $612 / M$ & $22 / 22$ & $0.2 / 0.2$ & NA \\
\hline PCG-30 & c. $-244 C>T$ & - & PCG/B & $\mathrm{O} / \mathrm{M}$ & $(23 / 28)$ & NA & Surgery \\
\hline PCG-105 & c. $-244 C>T$ & - & PCG/B & $3 / F$ & $(30 / 31.5)$ & $(0.6-0.7 / 0.7-0.8)$ & $2+$ Surgery \\
\hline PCG-96 & c. $-429 \mathrm{C}>\mathrm{G}(\mathrm{rs} 77888940)$ & - & PCG/B & $2 / \mathrm{M}$ & $(32 / 36)$ & $(0.2 / 0.2)$ & Surgery \\
\hline PCG-169 & c. $-429 \mathrm{C}>\mathrm{G}(\mathrm{rs} 77888940)$ & - & PCG/U (OD) & $\mathrm{NA} / \mathrm{F}$ & NA & NA & $2+$ Surgery \\
\hline PCG-54 & c.1134_144del & p.(G380Rfs*144) & PCG/B & $4 / \mathrm{M}$ & $(35 / 35)$ & $(0.6 / 0.7)$ & Surgery \\
\hline PCG-98 & c. ${ }^{*} 734 \mathrm{~A}>\mathrm{T}(\mathrm{rs} 35717904)$ & - & LCG/U (OD) & $60 / F$ & $(57 / 12)$ & $(0.6 / 0.4)$ & $1+$ Surgery \\
\hline PCG-84 (II:2) & $\begin{array}{c}\text { c.889C }>\text { T }(\text { FOXC } 1) \\
\text { c. }{ }^{454 C}>\mathrm{T}(\text { rs6533526) }(\text { PITX2) }\end{array}$ & $\begin{array}{l}\text { p.(P297S) } \\
(\text { FOXC1) }\end{array}$ & PCG/B & $6 / F$ & $15 / 18$ & NA & Surgery \\
\hline PCG-84 (III:1) & $\begin{array}{c}\text { c.889C }>\text { T }(\text { FOXC } 1) \\
\text { c. }{ }^{454 C}>\mathrm{T}(\text { rs6533526) }(\text { PITX2) }\end{array}$ & $\begin{array}{l}\text { p.(P297S) } \\
(\text { FOXC1) }\end{array}$ & Normalc $^{\mathrm{c}}$ & $24^{d}$ & - & - & NA \\
\hline
\end{tabular}

Abbreviations: LCG/PCG, late-onset primary congenital glaucoma/primary congenital glaucoma; B/U, bilateral/unilateral; C/D, cup/disk ratio; NA, not available; OH, ocular hypertension; OD/OS, right eye/left eye.

aThe variants were present in the heterozygous state and were named according to RefSeq NM_001453.2 and NM_00325.5 for FOXC1 and PITX2, respectively.

bNumber of drugs and/or surgery.

${ }^{\mathrm{C}}$ At the time of the study.

${ }^{\mathrm{d}}$ Age at the time of the study. 
a

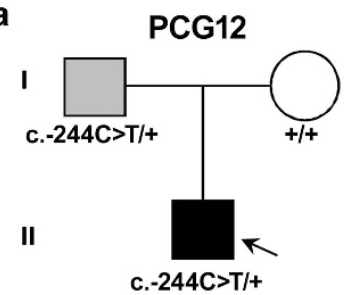

PCG14

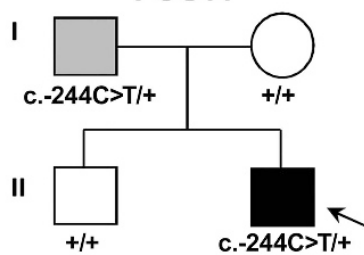

\section{c. $-244 C>T$}

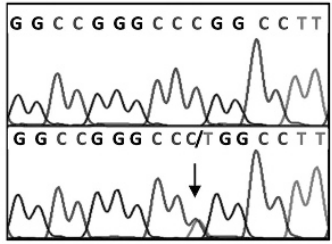

Wild type

(PCG14, I:2)

Mutant

(PCG14, II:2) b

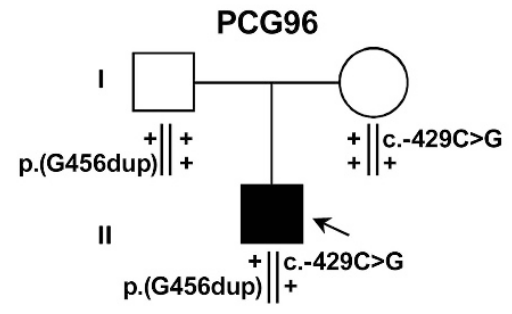

C

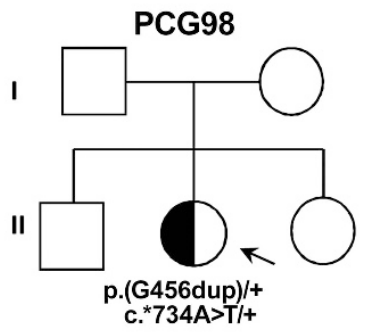

d

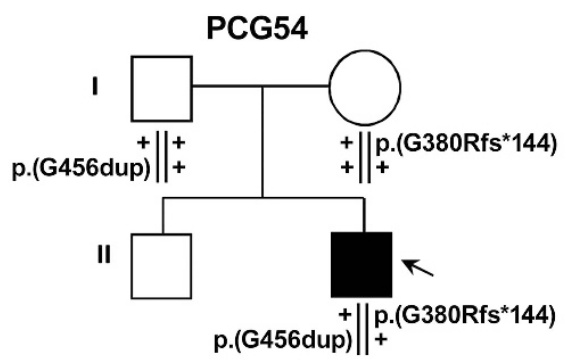

c. $-429 C>G$

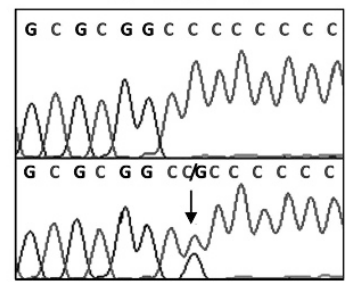

Wild type

(PCG96, I:1)

Mutant

(PCG96, II:1)

c. ${ }^{*} 734 \mathrm{~A}>\mathrm{T}$

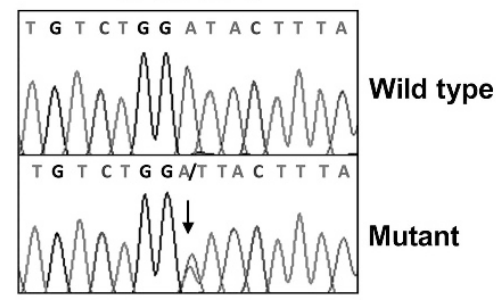

c.1134-1144del (p.(380Rfs*144))

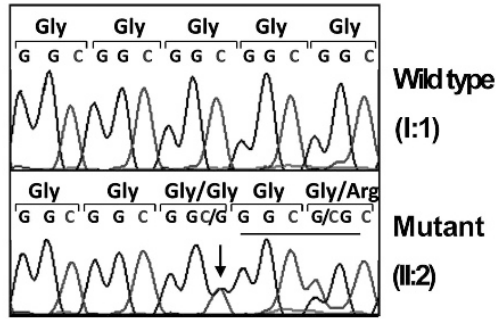

e PCG84

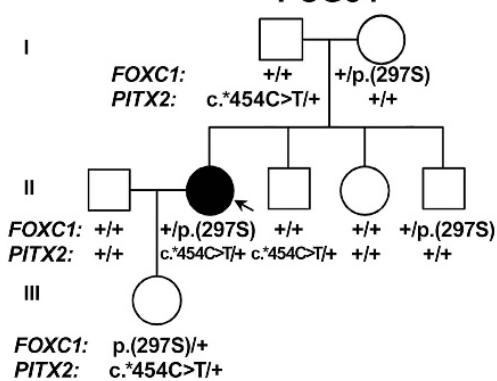

(FOXC1) c.889C>T (p.(P297S))

(PITX2)

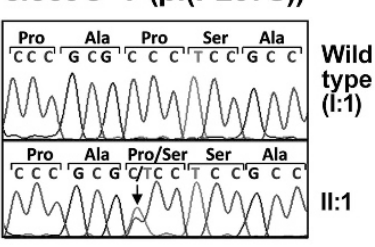
c. ${ }^{*} 454 \mathrm{C}>\mathrm{T}$

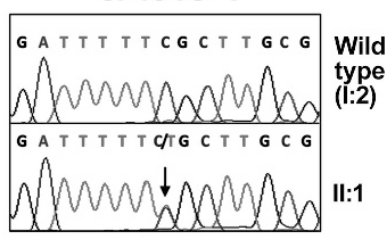

Figure 1 Segregation analysis of the FOXC1 variants identified in PCG families. Pedigree of the families who carry the rs $185790394: C>T$, C.-244C $>T$ (a), rs77888940:C > G, c.-429C > G (b), rs35717904:A>T, c. ${ }^{* 734 A>T ~(c), ~ p .(G 380 R f s * 144) ~(d) ~ a n d ~ r s 79691946: C ~>~ T, ~ p .(P 297 S) ~(e) ~ F O X C 1 ~ v a r i a n t s . ~}$ Segregation of the $c .{ }^{*} 454 C>T$ PITX2 variant is also shown in (e). The two vertical lines located below some of the pedigree symbols indicate haplotypes. Gray and black symbols indicate ocular hypertension and primary congenital glaucoma, respectively. The arrow in the pedigrees designates the index case. +: Wild-type allele. All of the variants were detected in the heterozygous state. The inset in each panel corresponds to representative electropherograms of the variants identified in each family. To facilitate comparison, the wild-type sequence is shown above the corresponding mutant sequence. Arrows in the insets indicate the location of variants. 
segment dysgenesis (ASD). ${ }^{14,15}$ According to the Ensembl variation database, c. ${ }^{\star} 454 \mathrm{C}>\mathrm{T}$ is present at low frequencies in African and American subjects (5\% and $2 \%$, respectively), and is absent in European and Asian individuals. It was not present in 233 Spanish control individuals (466 chromosomes).

Bioinformatics analysis and evolutionary conservation of variants Our analysis showed that the coding variants p.(P297S) and p. (G380Rfs $\left.{ }^{\star} 144\right)$ mapped at the inferred intrinsically disordered regions 2 and 3 (IDR2 and IDR3, respectively) of the inhibition domain (Figure 2a). The first of these variants affected an amino-acid residue situated in a Pro- and Ser-rich region (Figure 2a). The known variant p.(G456dup) localized at the Gly tract situated in the IDR4 (Figure $2 \mathrm{a}$ and $\mathrm{c}$ ). The two noncoding variants (c.-429C $>\mathrm{G}$ and c.-244C $>$ T), located in the $5^{\prime}$-UTR are predicted to alter a terminal olygopyrimidine tract (TOP) translation regulatory sequence and to increase the length of paired nucleotides in the mRNA secondary structure in this region, respectively (Figure 2b). Finally, the FOXC1 c. ${ }^{\star} 734 \mathrm{~A}>\mathrm{T}$ and PITX2 c. ${ }^{\star} 454 \mathrm{C}>\mathrm{T}$ variants were localized in the predicted seed target sequences for hsa-miR-548l and hsa-miR-548p, respectively (Figure $2 \mathrm{~b}$ and $\mathrm{d}$, respectively).

Evolutionary conservation analyses revealed that the coding variants affected amino acids conserved among mammals (Figure 2c). Interestingly, among the noncoding variants, the c.-429C nucleotide was present exclusively in the humans (Figure 2c), whereas olygopyrimidine tracts, similar to that in which the human variant was embedded, were observed in macaque and rat DNA sequences (Figure 2c). The PITX2 c. ${ }^{\star} 454$ cytosine was conserved in all analyzed mammal DNA sequences (Figure 2e).

\section{Transactivation activity assay}

The transactivation activity of the different FOXC1 variants was assessed by transient co-transfection of the reporter luciferase gene coupled to the CXCR4 promoter region that contains a FOX-binding element (FBE) (Figure 3a), with recombinant cDNA constructs encoding each FOXC1 variant (Figure $3 \mathrm{~b}$ and $\mathrm{d}$ ). Three mutants (p. $\left(\right.$ G380Rfs $\left.{ }^{\star} 144\right), \quad$ c. $-429 \mathrm{C}>\mathrm{G}$ and c. $\left.^{\star} 734 \mathrm{~A}>\mathrm{T}\right)$ showed both increased protein levels and transactivation activity of $\sim 130$ to $180 \%$ (Figure 3b-d) compared with wild-type, and therefore these variants were classified as hypermorphic alleles. Conversely, the p.(P297S) and c.-244C > T variants are hypomorphic alleles because they reduced transactivation by $\sim 50 \%$ (Figure $3 \mathrm{~b}$ and $\mathrm{c}$ ). The FOXC1 protein levels associated with c. $-244 \mathrm{C}>\mathrm{T}$ were lower than those of the 3'-UTR wild-type sequence. The lowest transactivation activity, however, was associated with the control p.(I126M) mutation (previously identified in patients with ARS and glaucoma ${ }^{6}$ ) that also presented with decreased protein levels (Figure 3b). Finally, the polymorphic p.(G456dup) variant also increased transactivation activity to $\sim 140 \%$ of the wild-type levels (Figure $3 \mathrm{~b}$ ). Western blot analyses of both RFP and LDH proteins showed no significant differences in either transfection efficiency or sample loading.

\section{miRNA evaluation}

The PITX2 c. ${ }^{\star} 454 \mathrm{C}>\mathrm{T}$ variant was predicted to be embedded in a target sequence for hsa-miR-548p (Supplementary Figure 1A). The functional effect of this nucleotide substitution was evaluated by parallel expression in cells in cultures of mutant or wild-type PITX2 $3^{\prime}$-UTRs, fused to the $3^{\prime}$-end of the luciferase coding sequence (Supplementary Figure 1B). Luciferase activity associated with the mutant sequence was $\sim 40 \%$ lower than that of the wild-type (Supplementary Figure 1C), suggesting that the variant increases the affinity of the target sequence for this endogenously expressed miRNA. Western blot analyses of both RFP and LDH proteins showed no significant differences in either transfection efficiency or sample loading.

\section{Protein stability}

The protein stability of the coding variants was assessed in transiently transfected cells treated with the protein synthesis inhibitor cycloheximide. Western blot analyses of both RFP and LDH proteins showed no significant differences in either transfection efficiency or sample loading within a time point (Figure 4a). The p.(P297S) variant was as stable as the wild-type protein (Figure $4 \mathrm{~b}$ ), with an estimated half-life of $12.37 \mathrm{~h}$ (Figure 4c). These data suggest that p.(P297S) alters transactivation activity independently of protein stability. In contrast, $8 \mathrm{~h}$ after cycloheximide treatment, the protein levels of the p.(G380Rfs $\left.{ }^{\star} 144\right)$ variant were almost $40 \%$ higher than those of the wild type (Figure $4 \mathrm{a}$ and $\mathrm{b}$ ), with an estimated 2.6-fold increase in its half-life (12.95 h vs $34.14 \mathrm{~h}$; Figure $4 \mathrm{c})$. The lowest protein stability corresponded to the p.(I126M) control mutant (4.20 h; Figure 4).

\section{DNA-binding ability and subcellular localization}

The DNA-binding ability of the coding FOXC1 variants was evaluated by electrophoretic mobility-shift assay (EMSA) analysis. These experiments showed the DNA-binding ability of the p.(P297S) and p.(G380Rfs $\left.{ }^{\star} 144\right)$ variants did not differ significantly from the wild type (Supplementary Figure 2A and B). In contrast, the intensity of the protein-DNA complexes formed by the $\mathrm{p}$.(I126M) control mutation were significantly lower than those formed by the wild-type protein (Supplementary Figure 2A and B).

Fluorescence immunocytochemistry of recombinant wild-type FOXC1 showed that the protein is localized exclusively in the nucleus (Supplementary Figure 3, wild type). The p.(I126M) control mutation and the other two coding variants showed normal subcellular nuclear localization (Supplementary Figure 3).

\section{DISCUSSION}

It is widely accepted that trabecular meshwork developmental abnormalities underlie the progression of PCG. ${ }^{16,17}$ Congenital glaucoma is frequently associated with severe functional alterations of FOXC1 that lead to ARS phenotypes. ${ }^{6}$ Therefore, we hypothesized that moderate functional alterations of the FOXC1 gene may contribute to relatively mild phenotypic alterations limited to congenital glaucoma. To test this hypothesis, we performed a comprehensive screening and a detailed functional analysis of FOXC1 variants in, as far as we know, the largest European cohort of PCG patients that contained 133 Spanish probands with no CYP1B1 or $M Y O C$ genetic alterations.

Presence of rare FOXC1 variants in congenital glaucoma patients In accordance with our working hypothesis, we identified rare heterozygous FOXC1 variants that were associated with moderate alterations in transactivation activity in patients with no known familial congenital glaucoma history. Because these variants were either not found or underrepresented in the control group, it is unlikely that they would be present in the patients merely by chance, and therefore these data suggest that the variants that we identified may contribute to nonmonogenic PCG inheritance, although this hypothesis does require additional confirmation studies. In addition, one of the carriers of a FOXC1 variant, diagnosed at the age of 6 months, PCG8-4 (Figure 1e), also coinherited a rare and hypomorphic PITX2 variant in the heterozygous state, further 
a
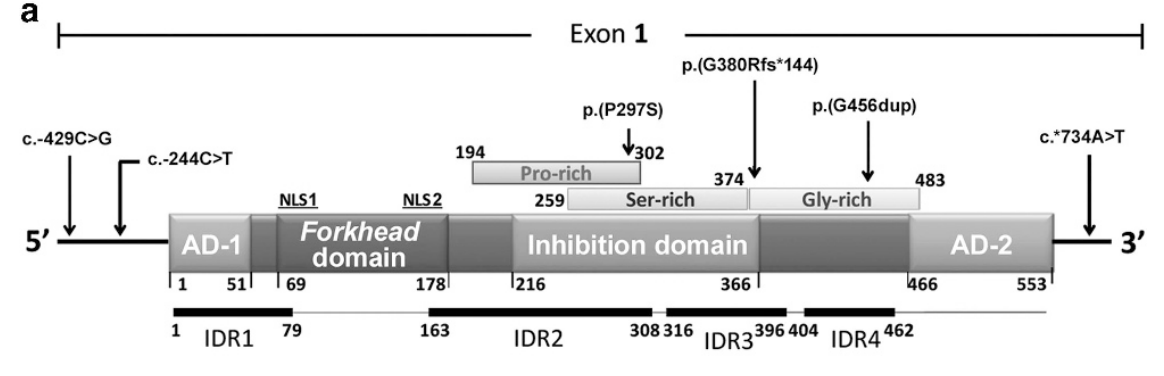

b

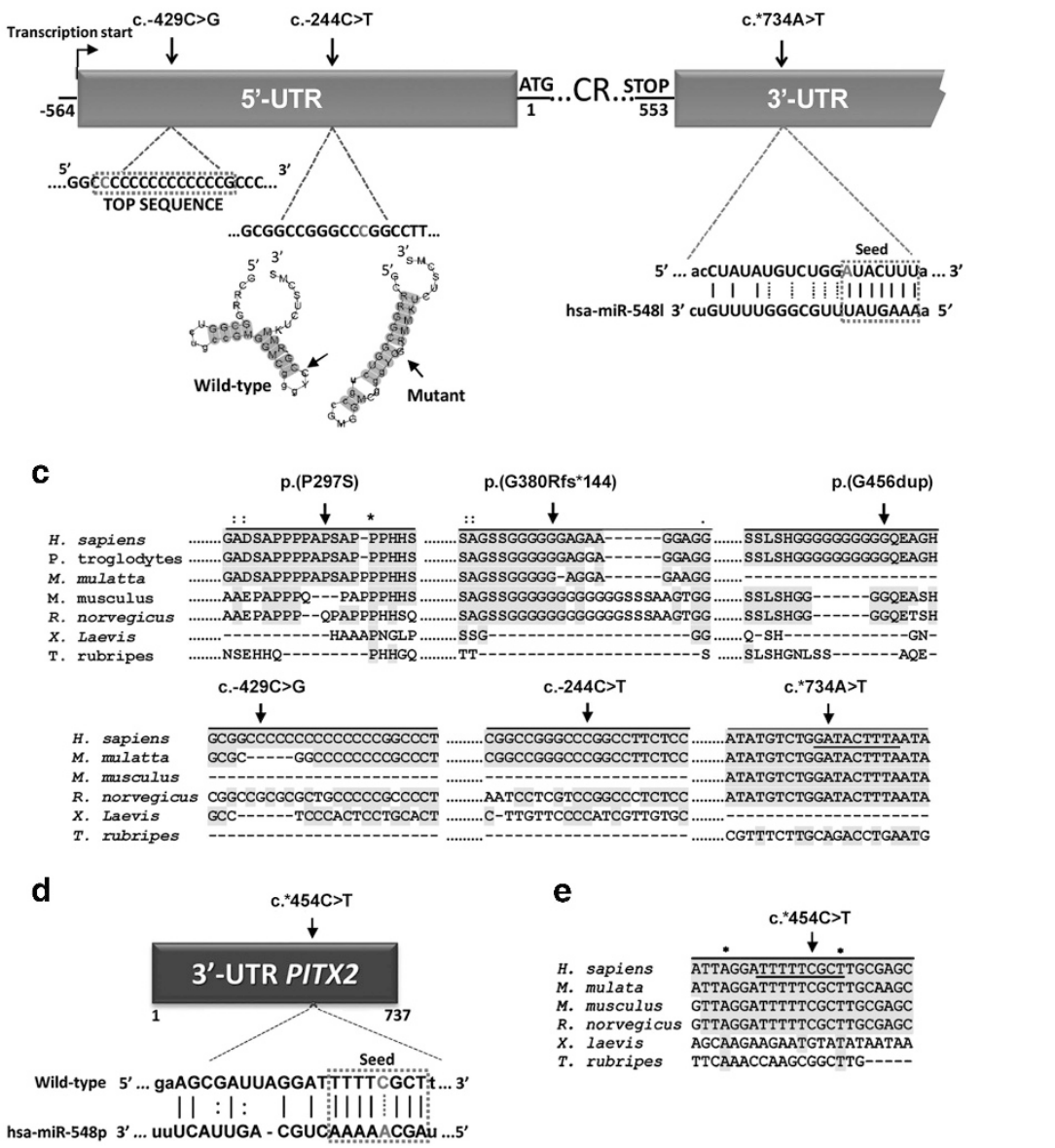

Figure 2 Localization of FOXC1 and PITX2 variants identified in this study. (a) Localization of the predicted and previously identified structural domains and motifs of FOXC1 polypeptide chain. The predicted Pro-, Ser- and Gly-rich regions reported in the Prosite database are shown above the scheme. Four intrinsically disordered regions (IDRs) inferred with the DisEMBL ${ }^{26}$ and Globplot $2^{27}$ programs are shown below the protein. $A D$, activation domain; $C R$, coding region; NLS, nuclear localization signal. (b) Localization of the predicted FOXC1 UTR regulatory sequences. The inference of functional RNA motifs in the 5'-UTR rsequence was carried out with RegRNA2 (http://regrna2.mbc.nctu.edu.tw/). The GeneBee-Molecular Biology Server (http://www.genebee.msu.su/ genebee.html) was used for RNA secondary structure prediction. The hsa-mir-548I target sequence was inferred with the MicroSNiPer software. The variable nucleotide is indicated in red. Vertical continuous and discontinuous lines in the miRNA target sequence indicate perfect Watson-Crick and G:U wobble pairings, respectively. Underlined nucleotides indicate the predicted seed target sequence for hsa-mir-548I. (c) Multiple alignments of variable amino-acid or nucleotide FOXC1 sequences. Both nucleotide and amino-acid sequence alignments were carried out with Clustal W. ${ }^{28}$ Arrows denote mutated positions. The asterisks indicate positions where all of the amino acids are identical, two vertical dots specify amino acids with similar chemical properties and one dot denotes amino-acid positions with weak chemical similarity. (d) Localization of the PITX2 c. ${ }^{*} 454 \mathrm{C}>\mathrm{T}$ variant in the predicted seed target sequence for hsamiR-548p. The variable nucleotide is indicated in red. The target miRNA was predicted with the MicroSNiPer software. The underlined nucleotides indicate the predicted seed target sequence for hsa-mir-548p. Vertical lines and dots in the predicted miRNA target sequence indicate perfect Watson-Crick and G:U wobble pairings, respectively. (e) Multiple alignments of the variable PITX2 nucleotide sequence. Nucleotide sequence alignment was carried out as indicated in (c). The symbols are the same as in (c). A full color version of this figure is available at the European Journal of Human Genetics journal online.

suggesting the existence of nonmonogenic PCG inheritance and a possible oligogenic transmission pattern involving these two genes. The patient transmitted both variants to her daughter who was 2 years old and phenotypically normal at the time of the study. Although we cannot rule out a delayed manifestation of the disease (variable expressivity), the current data suggest that the combination of only these two nucleotide changes is not enough to produce the disease, indicating a complex glaucoma inheritance. Follow-up of the infant is 


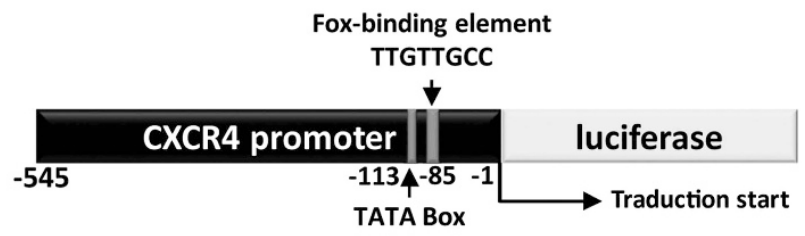

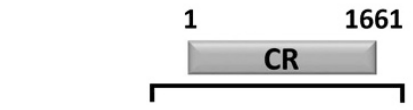

b

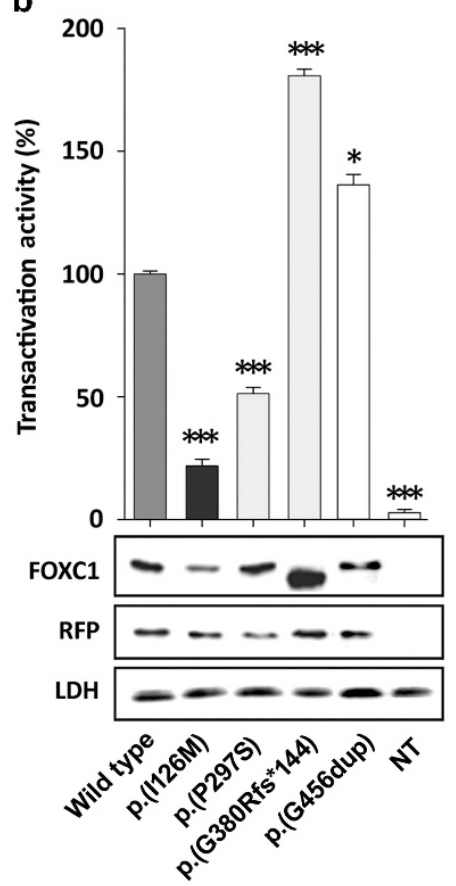

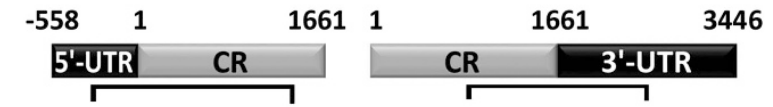

c

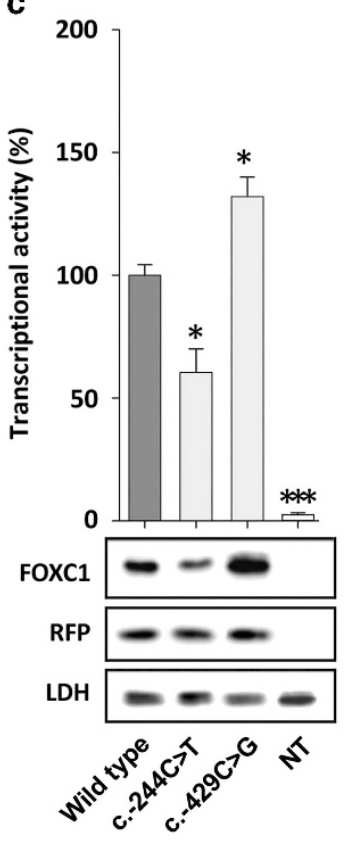

a

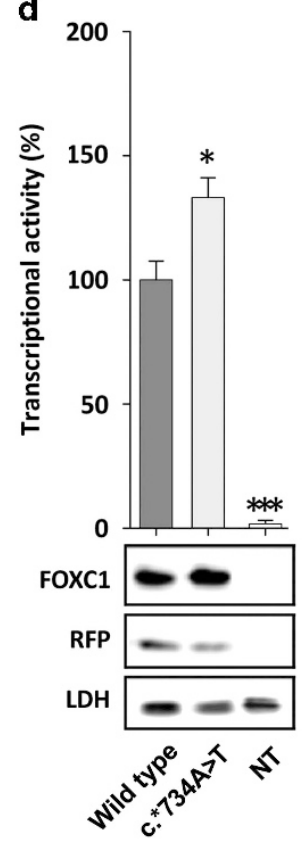

Figure 3 Altered transactivation activity of the FOXC1 variants identified in PCG patients. (a) A scheme of the cDNA construct containing the FOXC1-binding element present in the CXCR4 promoter, fused to luciferase and cloned into the PGL3 basic vector. This construct was used as a reporter of transactivation activity in co-transfection assays of the identified FOXC1 variants and the empty pMirTarget vector that encodes RFP (transfection efficiency control). The numbers below the scheme correspond to nucleotide positions. The cartoons above (b-d) indicate the FOXC1 mRNA regions incorporated into the different CDNA constructs that we used to assess the trancriptional activity of the different variants. CR, coding region. cDNA constructs encoding the indicated FOXC1 variants corresponding only to the coding region (b), the coding region with the $5^{\prime}$-UTR (c) or the coding region fused to the $3^{\prime}$-UTR (d) were transiently coexpressed with the reporter cDNA construct in HEK-293 T cells. The transcription activity of variants, expressed as a percentage of the luciferase activity of the wild-type protein, was measured as indicated in the Materials and methods section. The protein levels of the different FOXC1 versions present in HEK-293 T cells $48 \mathrm{~h}$ after transfection were determined by western immunoblot using a monoclonal anti-myc antibody (Santa Cruz Biotechnology). Each lane contained $15 \mu \mathrm{g}$ of the total protein obtained from the cell lysates. Transfection efficiency was assessed by western immunoblot using an anti-RFP antibody (Evrogen). The sample loading control, endogenous LDH, was also detected by immunoblot using an anti-LDH antibody (Chemicon). Error bars correspond to the SD of three independent experiments carried out in triplicate. Asterisks indicate statistically significant difference with respect to the control. The $P$-values were obtained using a one-way ANOVA followed by Tukey's multiple-comparison test: ${ }^{*} P<0.05$; ${ }^{* * *} P<0.001$. NT, nontransfected.

required to clarify the inheritance pattern in this family. Nonmonogenic PCG transmission has long been suspected based on the existence of incomplete penetrance and variable expressivity. In addition, it has been found that a significant proportion (close to $20 \%$ ) of PCG patients who carry CYP1B1 mutations inherited them in the heterozygous state. ${ }^{1}$ This finding led to the suggestion that PCG is not a simple Mendelian disease and that the action of modifier factors (genetic, stochastic and/or environmental) can significantly influence disease presentation. ${ }^{1,18}$ The possible existence of oligogenic glaucoma inheritance, caused by mutations in more than one gene, is not surprising because this disease is the result of abnormal development of the anterior eye segment, a complex phenomenon involving many intricate gene networks and molecular pathways. In accordance with the idea of oligogenic glaucoma, inheritance involving both CYP1B1 and MYOC has been reported in early-onset glaucoma ${ }^{19}$ and PCG. ${ }^{4}$ FOXC1/PITX2 glaucoma transmission has also been described in ARS. ${ }^{20}$

As noted above, our data also indicate a role for the moderately hypermorphic p.(G456dup) variant as a possible modifying factor. Interestingly, this variant was present in two patients who also had two other hypermorphic variants (c.-429C $>\mathrm{G}$ and p. (G380Rfs $\left.{ }^{\star} 144\right)$ in families PCG-96 and PCG-54, respectively) (Figure $1 \mathrm{~b}$ and d), contributing to the overall increase in FOXC1 activity associated with these genotypes. Moreover, we have reported that the p.(G456dup) variant coinherited in one patient along with another hypermorphic FOXC1 variant $\left(\mathrm{p} .\left(\mathrm{Y} 47^{*}\right)\right)$ seems to anticipate the glaucoma onset, ${ }^{21}$ 


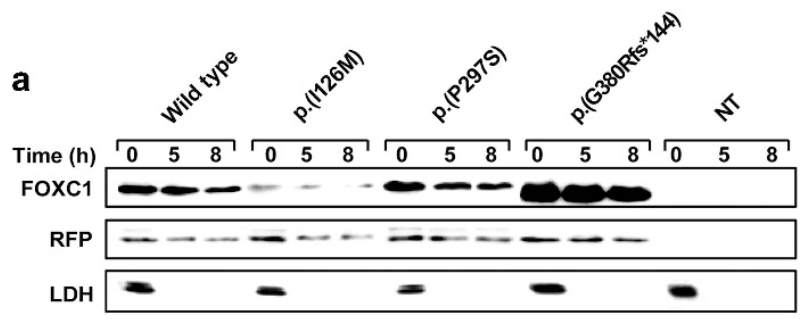

b
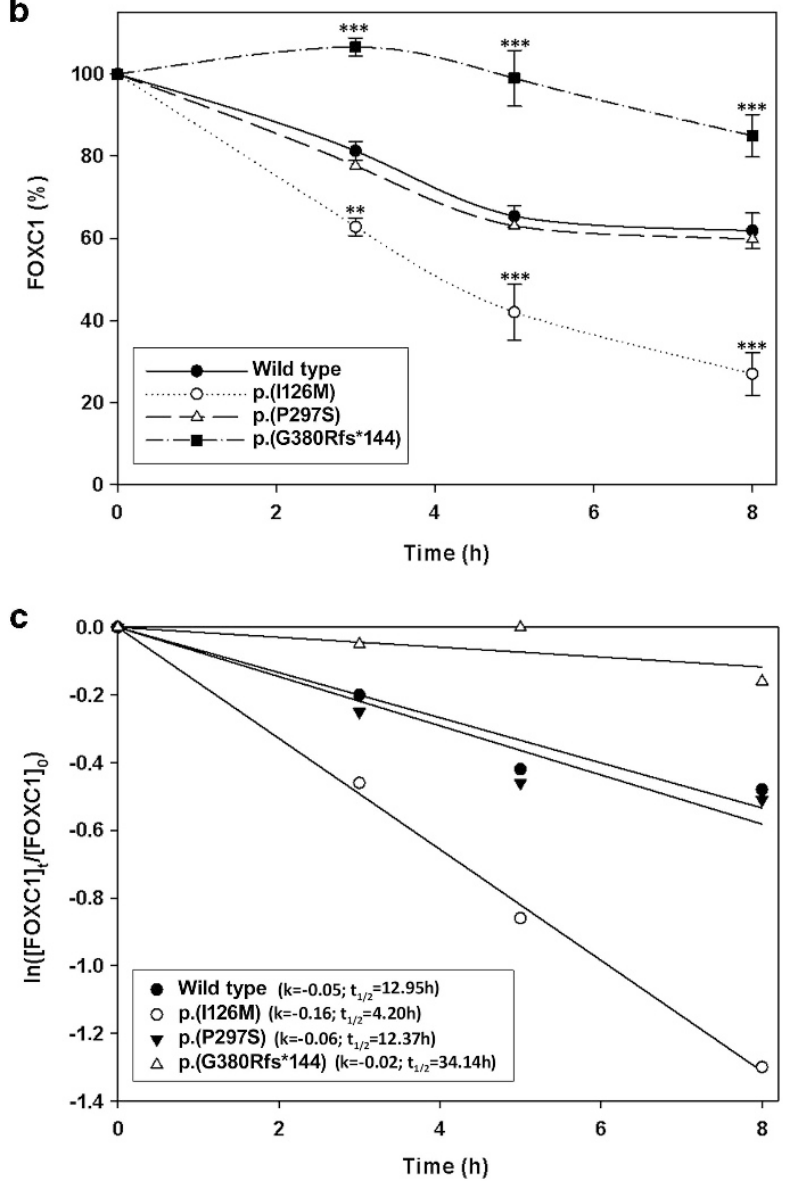

Figure 4 The FOXC1 p.(G380Rfs*144) variant alters protein stability. (a) Time-course stability analyses of FOXC1 mutant polypeptides found in PCG patients were carried out by transient expression of the corresponding cDNA constructs in HEK-293 T cells. The transfected cells were treated with the protein synthesis inhibitor cycloheximide and the different recombinant FOXC1 variants were detected by western immunoblot using an anti-myc monoclonal antibody (Santa Cruz Biotechnology) at the indicated time points. Transfection efficiency was assessed by co-transfection with the nonrecombinant pMirTarget vector that encodes RFP. RFP was detected by western blot using an antiRFP antibody (Evrogen). The sample loading control, endogenous LDH, was also detected by immunoblot using an anti-LDH antibody (Chemicon). (b) The amount of FOXC1 at the indicated time points was determined by densitometry analysis of the signals detected via western blot. Relative amounts of FOXC1 are expressed as a percentage of the protein levels at time $0 \mathrm{~h}$. (c). The rate of decay $(k)$ and half-lives $\left(t_{1 / 2}\right)$ of the recombinant FOXC1 variants at the indicated time points was determined using a linear regression analysis as described in the Materials and methods section. The error bars correspond to the SD of three independent experiments carried out in triplicate. The asterisks indicate statistically significant differences with respect to the control. The $P$-values were obtained using a one-way ANOVA followed by Tukey's multiplecomparison test: ${ }^{*} P<0.01 ;{ }^{* *} P<0.001$. further supporting its role as a modifier factor. We have observed that the p.(G456dup) variant moderately increases transactivation independent of protein stability and DNA-binding ability (unpublished data). As shown previously, p.(G456dup) is located in a Gly-rich region within an IDR of the protein that is important for molecular interactions. Therefore, the length of this region could affect proteinprotein interactions and modulate the transactivation activity of FOXC1.

\section{Functional evaluation of the rare variants and identification of} regulatory sequences

Three of the five identified variants mapped at UTRs and two affected the coding region of FOXC1. The c. $-429 \mathrm{C}>\mathrm{G}$ purine/pyrimidine transversion was predicted to impair a putative TOP regulatory motif that is known to coordinate translation repression of vertebrate ribosomal proteins and translation elongation factors. ${ }^{22}$ This motif consists of 5-15 pyrimidines, beginning with a $\mathrm{C}$ residue and ending in a $G$ nucleotide and, remarkably, it has been reported that the exchange of purines for pyrimidines in this motif results in unregulated translation. ${ }^{22}$ According to these data, the simultaneous increase in transactivation and FOXC1 protein levels associated with the c. $-429 \mathrm{C}>\mathrm{G}$ variant supports the idea that the mutant has a diminished ability to repress mRNA translation. The second $5^{\prime}$-UTR variant that we found, c. $-244 \mathrm{C}>\mathrm{T}$, was predicted to alter the mRNA secondary structure in this region. It is known that the $5^{\prime}$-UTR secondary structure influences mRNA translation, ${ }^{23}$ and thus this variant has the potential to impair mRNA binding to regulators of protein synthesis. With regard to the ${ }^{\star} .734 \mathrm{~A}>\mathrm{T}$ variant, we have observed that it disrupts the hsa-miR5481 target sequence, leading to improved mRNA stability and thus to increased protein and transactivation levels. ${ }^{24}$ Overall, our results support the hypothesis that these noncoding variants affect function. Moreover, the functional analyses of these variants provide the first evidence for the existence of translation negative cis-regulatory elements in both the $5^{\prime}$ - and $3^{\prime}$-UTR FOXC1 sequences and whose impairment likely participates in glaucoma. The p. (G380Rfs $\left.{ }^{\star} 144\right)$ variant generated a hypermorphic and truncated polypeptide lacking the C-terminal region that normally participates in FOXC1 degradation via the ubiquitin $26 \mathrm{~S}$ proteasome pathway..$^{10}$ According to these data, p. $\left(\right.$ G380Rfs $\left.{ }^{\star} 144\right)$ showed remarkably increased protein stability that is likely the basis for its high transactivation activity.

Functional evaluation of the two gene variants identified in the patient with putative FOXC1/PITX2 glaucoma inheritance showed that the reduced transactivation activity of FOXC1 p.(P297S) does not depend on altered protein stability, DNA binding or nuclear localization. However, the fact that this variant maps to the predicted IDR3 opens the possibility that it interferes with the interactions of the transcription factor with other regulatory proteins, as discussed previously. In accordance with our results, it has been reported that p.(P297S) (1) shows reduced transactivation activity, (2) is associated with anterior segment digenesis and (3) is likely to disrupt FOXC1 interactions with other unidentified factors involved in transactivation. ${ }^{14}$ In contrast to our results, however, a previous study has reported a moderately extended half-life for the p.(P297S) variant (45\% longer than wild type). ${ }^{14}$ The second variant discussed, PITX2 c. ${ }^{*} 454 \mathrm{C}>\mathrm{T}$, may increase the binding affinity of hsa-miR-548p to the mutant mRNA, leading to lower PITX2 protein levels. Interestingly, this miRNA belongs to the same family as hsa-miR-548l that regulates FOXC1 mRNA. ${ }^{24}$ Thus, coinheritance of rare FOXC1 and PITX2 variants could contribute to glaucoma development by a simultaneous moderate alteration in translation of two related transcription factors. 
Previous reports have suggested the existence of an upper and lower critical FOXC1 activity threshold associated with different phenotypes. ${ }^{25}$ In accordance with these reports, our data suggest that moderate functional alterations of FOXC1, along with other genetic and/or environmental factors, may act as a modifier factor and contribute to the expression of relatively mild goniodysgenesis that results in congenital glaucoma.

In summary, this study supports the existence of novel cisregulatory signals involved in FOXC1 translation and shows the existence of moderately altered rare variants of FOXC1 that may act as modifier factors in patients with PCG, suggesting the possible contribution of this gene to complex congenital glaucoma inheritance.

\section{CONFLICT OF INTEREST}

The authors declare no conflict of interest.

\section{ACKNOWLEDGEMENTS}

This study has been supported by research grants from the Regional Ministry of Health (GCS-2006_C/12) and the 'Instituto de Salud Carlos III' (RD07/0062/ 0014, RD12/0034/0003 and PI11/00662). M García-Antón is the recipient of a fellowship from the 'Ministerio de Educación, Cultura y Deporte' (FPU 13/03308). We thank Dr Aranzazu Díaz-de-Bustamante for providing samples from family PCG-84.

1 Lopez-Garrido MP, Medina-Trillo C, Morales-Fernandez L et al: Null CYP1B1 genotypes in primary congenital and nondominant juvenile glaucoma. Ophthalmology 2013; 120 716-723.

2 Ali M, McKibbin M, Booth A et al: Null mutations in LTBP2 cause primary congenital glaucoma. Am J Hum Genet 2009; 84: 664-671.

3 Chakrabarti S, Kaur K, Komatireddy $\mathrm{S}$ et al: Gln48His is the prevalent myocilin mutation in primary open angle and primary congenital glaucoma phenotypes in India. Mol Vis 2005; 11: 111-113.

4 Kaur K, Reddy AB, Mukhopadhyay A et al: Myocilin gene implicated in primary congenital glaucoma. Clin Genet 2005; 67: 335-340.

5 Chakrabarti S, Kaur K, Rao KN et al: The transcription factor gene FOXC1 exhibits a limited role in primary congenital glaucoma. Invest Ophthalmol Vis Sci 2008; 50: 75-83.

6 Nishimura DY, Swiderski RE, Alward WL et al: The forkhead transcription factor gene FKHL7 is responsible for glaucoma phenotypes which map to 6p25. Nat Genet 1998; 19: 140-147.

7 Lines MA, Kozlowski K, Walter MA: Molecular genetics of Axenfeld-Rieger malformations. Hum Mol Genet 2002; 11: 1177-1184.

8 Campos-Mollo E, Sanchez-Sanchez F, Lopez-Garrido MP, Lopez-Sanchez E, LopezMartinez F, Escribano J: MYOC gene mutations in Spanish patients with autosomal dominant primary open-angle glaucoma: a founder effect in southeast Spain. Mol Vis 2007; 13: 1666-1673.

9 Campos-Mollo E, Lopez-Garrido MP, Blanco-Marchite C et al: CYP1B1 gene mutations in Spanish patients with primary congenital glaucoma: phenotypic and functional variability. Mol Vis 2009; 15: 417-431.

10 Berry FB, Lines MA, Oas JM et al: Functional interactions between FOXC1 and PITX2 underlie the sensitivity to FOXC1 gene dose in Axenfeld-Rieger syndrome and anterior segment dysgenesis. Hum Mol Genet 2006; 15: 905-919.

11 Aroca-Aguilar JD, Martínez-Redondo F, Martín-Gil A, Pintor J, Coca-Prados M, Escribano J: Bicarbonate-dependent secretion and proteolytic processing of recombinant myocilin. PLoS One 2013; 8: e54385.

12 Mears AJ, Jordan T, Mirzayans F et al: Mutations of the forkhead/winged-helix gene, FKHL7, in patients with Axenfeld-Rieger anomaly. Am J Hum Genet 1998; 63: 1316-1328.

13 Stenson PD, Mort M, Ball EV, Shaw K, Phillips A, Cooper DN: The Human Gene Mutation Database: building a comprehensive mutation repository for clinical and molecular genetics, diagnostic testing and personalized genomic medicine. Hum Genet 2014; 133: 1-9

14 Fetterman CD, Mirzayans F, Walter MA: Characterization of a novel FOXC1 mutation, P297S, identified in two individuals with anterior segment dysgenesis. Clin Genet 2009; 76: 296-299.

15 Kaur K, Ragge NK, Ragoussis J: Molecular analysis of FOXC1 in subjects presenting with severe developmental eye anomalies. Mol Vis 2009; 15: 1366-1373.

16 Anderson DR: The development of the trabecular meshwork and its abnormality in primary infantile glaucoma. Trans Am Ophthalmol Soc 1981; 79: 458-485.

17 Sampaolesi R, Zarate J, Sampaolesi JR. Primary congenital glaucoma. In: The Glaucomas Volume 1 - Pediatric Glaucomas. Springer-Verlag: Heidelberg, 2009, pp 1-7.

18 Campos-Mollo E, Lopez-Garrido MP, Blanco-Marchite C et al: CYP1B1 mutations in Spanish patients with primary congenital glaucoma: phenotypic and functional variability. Mol Vis 2009; 15: 417-431.

19 Vincent AL, Billingsley G, Buys Y et al: Digenic inheritance of early-onset glaucoma: CYP1B1, a potential modifier gene. Am J Hum Genet 2002; 70: 448-460.

20 Kelberman D, Islam L, Holder SE et al: Digenic inheritance of mutations in FOXC1 and PITX2 : correlating transcription factor function and Axenfeld-Rieger disease severity. Hum Mutat 2011; 32: 1144-1152.

21 Medina-Trillo C, Sanchez-Sanchez F, Aroca-Aguilar J-D et al: Hypo- and hypermorphic FOXC1 mutations in dominant glaucoma: transactivation and phenotypic variability. PLoS One 2015; 10: e0119272.

22 Levy S, Avni D, Hariharan N, Perry RP, Meyuhas O: Oligopyrimidine tract at the $5^{\prime}$ end of mammalian ribosomal protein mRNAs is required for their translational control. Proc Natl Acad Sci USA 1991; 88: 3319-3323.

23 Chatterjee S, Pal JK: Role of 5'- and 3'-untranslated regions of mRNAs in human diseases. Biol Cell 2009; 101: 251-262.

24 Medina-Trillo C, Aroca-Aguilar JD, Ferre-Fernandez JJ et al: The role of hsa-mir-548| dysregulation as a putative modifier factor for glaucoma-associated FOXC1 mutations. Microrna 2015; e-pub ahead of print 20 March 2015.

25 Walter MA: PITs and FOXes in ocular genetics: the Cogan lecture. Invest Ophthalmol Vis Sci 2003; 44: 1402-1405.

26 Linding R, Jensen LJ, Diella F, Bork P, Gibson TJ, Russell RB: Protein disorder prediction: implications for structural proteomics. Structure 2003; 11: 1453-1459.

27 Linding R, Russell RB, Neduva V, Gibson TJ: GlobPlot: exploring protein sequences for globularity and disorder. Nucleic Acids Res 2003; 31: 3701-3708.

28 Thompson JD, Higgins DG, Gibson TJ: CLUSTAL W: improving the sensitivity of progressive multiple sequence alignment through sequence weighting, position-specific gap penalties and weight matrix choice. Nucleic Acids Res 1994; 22: 4673-4680.

Supplementary Information accompanies this paper on European Journal of Human Genetics website (http://www.nature.com/ejhg) 